\title{
Curvas de desempenho de válvulas reguladoras de pressão novas e com diferentes tempos de utilização ${ }^{1}$
}

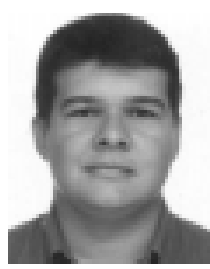

\author{
Sílvio C. R. V. Lima ${ }^{2}$, José A. Frizzone ${ }^{3}$, Raimundo N. T. Costa ${ }^{4}$, Francisco de Souza ${ }^{5}$, \\ Anderson S. Pereira ${ }^{6}$, Carmelo C. Machado ${ }^{7} \&$ Manoel Valnir Júnior ${ }^{8}$ \\ 1 Parte da Dissertação de Mestrado do primeiro autor apresentada à Universidade Federal do Ceará \\ 2 CENTEC. Av. Dr. Guarani 317, CEP 62040-730, Sobral, CE. E-mail: silviocarlos@centec.org.br (Foto) \\ ${ }^{3}$ ESALQ/USP. Av. Pádua Dias 11, CP 9, CEP 13418-900, Piracicaba, SP. E-mail: frizzone@carpa.ciagri.usp.br \\ ${ }^{4}$ DENA/UFC. Campus do Pici, Bloco 804, CEP 60455-760, Fortaleza, CE. Fone: (85) 288-9765, Fax: (85) $288-9756$.
} E-mail: rntcosta@ufc.br

5 DENA/UFC. E-mail: fsouza@ufc.br

${ }^{6}$ ESALQ/USP

${ }^{7}$ Doutorando. ESALQ/USP

8 CENTEC. E-mail: valnirjunior@ig.com.br

Protocolo $1-4 / 1 / 2002$ - Aprovado em 17/6/2003

Resumo: O desempenho de uma válvula reguladora de pressão, fabricada pela Fabrimar, Modelo Exact-20-Ref. RP-3-20-3/4"FF, utilizada em pivô central, foi avaliado em laboratório, conforme as normas ISO (1993). Este trabalho foi desenvolvido no Laboratório de Irrigação do Departamento de Engenharia Rural, ESALQ/USP, utilizando-se reguladores de pressão novos e com diferentes tempos de emprego no campo. As curvas de desempenho das válvulas novas satisfizeram as condições exigidas pela norma, caracterizando o regulador como do tipo A, para as velocidades de referência de $0,5 \mathrm{~m} \mathrm{~s}^{-1}$ (vazão de $0,57 \mathrm{~m}^{3} \mathrm{~h}^{-1}$ ) até $4,0 \mathrm{~m} \mathrm{~s}^{-1}$ (vazão de 4,50 $\mathrm{m}^{3} \mathrm{~h}^{-1}$ ) e várias pressões de entrada, sendo também elaborado um modelo estatístico para a pressão de saída do regulador novo, com uma vazão e uma pressão de entrada. Foram coletadas, de diversos sistemas tipo pivô central e de diferentes vãos, válvulas reguladoras de pressão do mesmo modelo, que possuíam tempos de uso de 2000, 2500, 6000, 8500, 9000 e 10000 h e, também, com diferentes qualidades de água utilizadas. Os ensaios seguiram os mesmos padrões dos novos, realizando-se uma análise estatística dos dados. Apesar dos desgastes provocados pela água de má qualidade e dos vazamentos apresentados, o desempenho hidráulico das válvulas com tempo de uso até $6000 \mathrm{~h}$, não diferiu do desempenho das novas, para pressões de entrada até 826,3 $\mathrm{kPa}$ e velocidades de referência até $2,5 \mathrm{~m} \mathrm{~s}^{-1}$. O desempenho dos reguladores de pressão com tempos de uso iguais ou superiores a $8500 \mathrm{~h}$, não se manteve semelhante ao do regulador novo, especialmente para pressões de entrada superiores a 481,7 $\mathrm{kPa}$.

Palavras-chave: reguladores de pressão, desempenho, pivô central

\section{Performance curves of new and used pressure regulating valves}

\begin{abstract}
The performance of a pressure regulator valve, manufactured by Fabrimar (Model Exact20-Ref. RP-3-20-3/4"FF), for central pivot applications, was evaluated in the laboratory following the ISO 1993 norms. The tests were conducted in the Laboratory of Irrigation of the Department of Rural Engineering, ESALQ/USP. The performance curves of the new valves satisfied the conditions established by the norm, characterizing the regulator as type A, for reference velocity of $0.5 \mathrm{~m} \mathrm{~s}^{-1}$ (flow of $0.57 \mathrm{~m}^{3} \mathrm{~h}^{-1}$ ) up to $4.0 \mathrm{~m} \mathrm{~s}^{-1}$ (flow of $4.50 \mathrm{~m}^{3} \mathrm{~h}^{-1}$ ) and several inlet pressures. A model for the outlet pressure of the new regulator, as a function of flow and inlet pressure, was also developed. Pressure regulators of a given model were collected from several central pivot systems and from different spans in the system, such that an array of pressure regulators with 2000, 2500, $6000,8500,9000$ and $10000 \mathrm{~h}$ of operation at several water qualities were obtained. These pressure regulator were evaluated according to ISO 1993 as well. In spite of the effect of water sediments on the pressure regulator and observed leaks, the hydraulic performance of the valves with $6000 \mathrm{~h}$ did not differ in comparison to the new ones, for inlet pressures of $826.3 \mathrm{kPa}$ and reference velocities up to $2.5 \mathrm{~m} \mathrm{~s}^{-1}$. The performance of the pressure regulators with $8500 \mathrm{~h}$ or more of operation did not perform as well as the new ones, especially for upper inlet pressures over $481,7 \mathrm{kPa}$.
\end{abstract}

Key words: pressure regulators, performance, center pivot 


\section{INTRODUÇÃO}

A inserção da irrigação no processo de produção agrícola é uma decisão tomada com base na probabilidade de se atingir um nível de eficácia na aplicação dessa técnica. A eficácia da irrigação é algo que se identifica pela relação custo-benefício e cuja maximização é função de uma série de fatores que vão desde as condições de mercado para os produtos agrícolas, até as características de desempenho dos emissores de água. Devido ao avanço tecnológico, os processos de tomada de decisão se tornam mais dependentes de dados e informações sobre constantes construtivas, especificações e parâmetros de desempenho, cuja obtenção constitui o objetivo fundamental dos ensaios dos equipamentos (Frizzone \& Botrel, 1996).

Os sistemas de irrigação precisam ser bem projetados, principalmente em áreas de topografia acidentada, onde ocorre uma variação acentuada na pressão do sistema, causando significativas diferenças de vazão, reduzindo a uniformidade de distribuição de água e a conseqüente diminuição da eficiência de aplicação, além de aumentar as perdas de água por escoamento superficial.

Para o bom funcionamento de um sistema de irrigação pressurizado, a alta uniformidade de distribuição de água entre os emissores é de grande importância, podendo ser influenciada pela combinação de diversos fatores, entre eles a variação de vazão dos emissores de água ao longo da linha lateral, em decorrência da variação de pressão e da própria variabilidade dos emissores, das condições de vento e da altura do emissor em relação ao solo (Frizzone, 1992). A variação de pressão ao longo da lateral é consequiência da perda de carga e da variação de nível, que são significativas nos sistemas de irrigação tipo pivô central, pela sua grande extensão.

Como alternativa para minimizar os efeitos da variação de pressão na vazão dos emissores em sistemas pivô central, utilizam-se válvulas reguladoras de pressão, que permitem a regulação da pressão de saída, numa faixa definida de pressão de entrada e vazão. A caracterização hidráulica desse tipo de válvula é feita pela curva de desempenho que relaciona a pressão de saída em função da pressão de entrada e da vazão. De acordo com Zaggo et al.(1990) as válvulas reguladoras de pressão são dispositivos que reagem a um acréscimo de pressão de entrada (ou de saída, dependendo do tipo de válvula) diminuindo a seção de passagem de água e aumentando a perda de carga localizada que ocorre na válvula, de forma a compensar o aumento da pressão de entrada, mantendo a pressão de saída dentro de certos limites que irão caracterizar a "pressão de regulação da válvula". A vazão do emissor depende do diâmetro do bocal e da pressão de operação, e esta é função da pressão de saída na válvula. A pressão de entrada na válvula, por sua vez, está relacionada com a pressão disponível na tubulação que depende da combinação linear entre perda de carga e diferença de nível. A pressão em um emissor pode variar por fatores hidráulicos ou diferenças de nível. Um regulador ideal deve manter constante a pressão de saída, independentemente da pressão de entrada e da vazão (Von Bernuth \& Baird, 1990). Klar et al. (2001) avaliaram o desempenho de reguladores novos e usados instalados em dois pivôs e observaram que após a troca dos kits compostos de difusores e reguladores de pressão, os CUC's aumentaram em 24,22 e 34,76\% e as lâminas aplicadas foram inferiores em 9,2 e 25,06\%, nos pivôs 1 e 2, respectivamente. Segundo Benami \& Ofen (1984) os reguladores são utilizados para obter a uniformidade de aplicação de água dentro de limites razoáveis para todos os aspersores em operação no campo. De acordo com Keller e Bliesner (1990) a dependência da vazão dos reguladores de pressão deveria ser incorporada no projeto de um sistema de irrigação tipo pivô central e, deste modo, os efeitos de vazões diferentes decorrentes da pressão de saída poderiam ser compensados, ajustando-se o diâmetro do bocal do aspersor.

Tarjuelo (1995) cita que os reguladores mais comuns são os de mola, constituídos por uma carcaça geralmente de polipropileno ou latão, que aloja um êmbolo. O êmbolo é empurrado por uma mola que tende a mantê-lo na posição de máxima abertura e, quando a pressão existente na água abaixo do regulador atua sobre a superfície do fixador da mola e essa pressão é menor que a do regulador, a mola mantém aberto o êmbolo e a água passa apenas com uma pequena perda de carga; mas quando esta pressão atuante é maior, ela se transmite momentaneamente, criando uma força que vence a da mola e o êmbolo se fecha parcialmente, aumentando a perda de carga até conseguir que a pressão fique próxima à do regulador.

A pressão de saída no regulador nem sempre é a descrita pelo fabricante, pois pode variar se a pressão de entrada e a vazão no sistema não corresponderem às apresentadas na curva de desempenho do regulador de pressão, induzindo o projetista a incorrer em erro no dimensionamento da vazão do emissor.

De acordo com a ISO (1993) o nível de exatidão de um regulador de pressão é dito $\mathrm{A}$ ou $\mathrm{B}$, de acordo com o valor do desvio da pressão de saída (pressão regulada) em relação à pressão declarada de regulação pelo fabricante, isto é:

$$
\text { Desvio }=\frac{P_{S}-P_{D}}{P_{D}} \times 100
$$

em que:

$\mathrm{P}_{\mathrm{S}} \quad$ - valor médio da pressão de saída regulada para a amostra

$\mathrm{P}_{\mathrm{D}}$ - pressão declarada de regulação.

Um regulador de pressão tem nível de exatidão A se o desvio não é superior a $10 \%$, e B, se não for superior a $20 \%$.

$\mathrm{O}$ intervalo de regulação da pressão de entrada é aquele que inclui a pressão regulada dentro do nível de exatidão especificado (regulador de pressão com nível de exatidão A: PD 10\%; regulador de pressão com nível de exatidão B: PD $20 \%$ ). A pressão inicial de regulação corresponde ao menor valor de pressão de entrada para o qual se obtém uma pressão regulada dentro do nível de exatidão especificado.

Como o equipamento nas condições de campo sofre ações de fatores climáticos e está exposto a danos mecânicos, é importante, também, se conhecer parâmetros que caracterizam a durabilidade da válvula reguladora de pressão em função do tempo de uso, comparando-se seu desempenho com peças novas. 
O objetivo deste trabalho foi ensaiar reguladores de pressão da marca Fabrimar, novos e com diferentes tempos de uso, avaliando-se as curvas de desempenho, em conformidade com as normas ISO - International Organization for Standardization - 10522 de 1993.

\section{MATERIAL E MÉTODOS}

Realizou-se o experimento no Laboratório de Irrigação da Escola Superior de Agricultura Luiz de Queiroz da Universidade de São Paulo, localizado em Piracicaba, SP. Utilizou-se a Norma ISO (1993) (Agricultural Irrigation Equipment - Direct-Acting Pressure - Regulating Valves), determinando-se as pressões de saída na válvula, em função das pressões de entrada e da vazão.

Utilizaram-se peças novas e usadas de um regulador de pressão fabricado pela Fabrimar S.A. Indústria e Comércio, Modelo EXACT-20 - 3/4" FF, nas quantidades de 20 peças novas e 25 peças usadas de cada pivô central amostrado, sendo estas agrupadas em amostras de 5 válvulas pertencentes a vãos onde operavam com a mesma vazão. As características declaradas são: pressão de regulação de 137,0 kPa (20 PSI) e diâmetro nominal $20 \mathrm{~mm}(3 / 4$ ").

Os reguladores usados, obtidos de diversos sistemas pivô central, em diferentes vãos, possuíam tempos de uso de 2000, 2500, 6000, 8500, 9000 e $10000 \mathrm{~h}$. Na Figura 1 pode-se observar um esquema desta válvula.

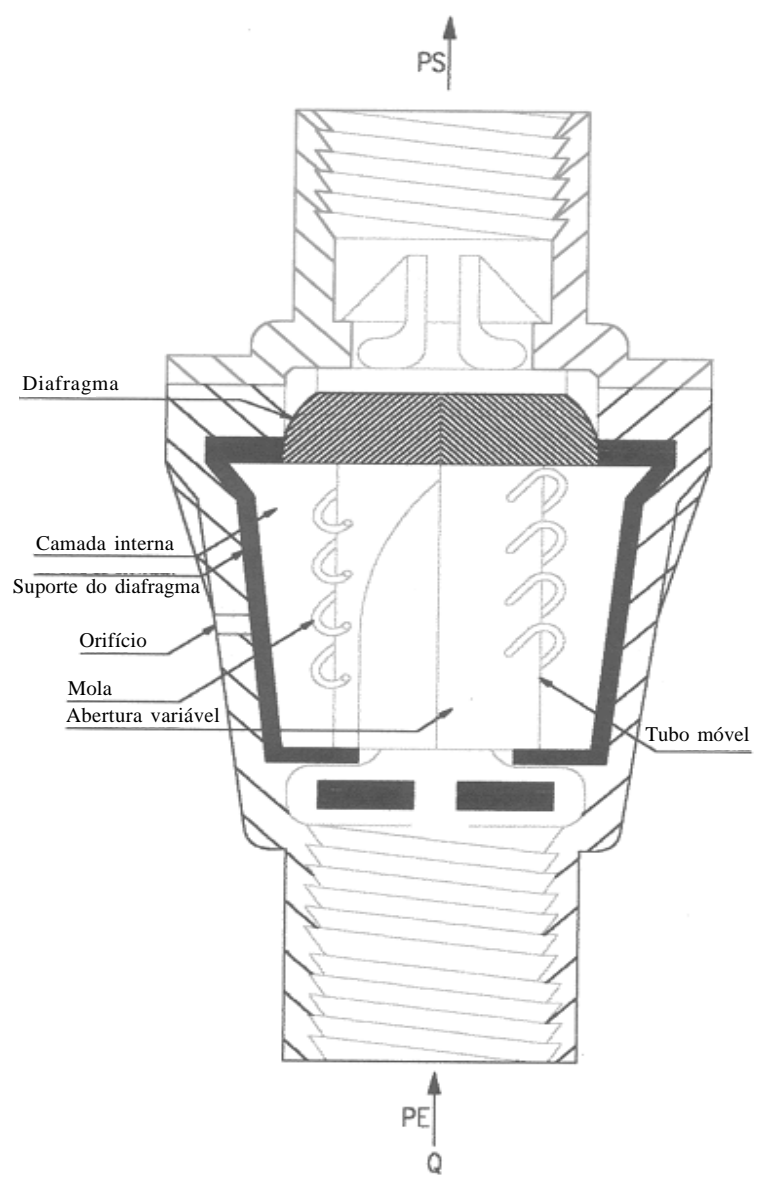

Figura 1. Esquema de uma válvula reguladora de pressão Fabrimar Modelo EXACT-20 - 3/4" FF
Os ensaios foram realizados em uma bancada, cujo circuito hidráulico era similar ao esquema apresentado no texto da norma ISO (1993) determinando-se as pressões de saída no regulador, em função das pressões de entrada, em diferentes vazões controladas. Com esses dados, construíram-se as curvas de desempenho dos reguladores de pressão.

A bancada de ensaio, apresentada na Figura 2, era constituída dos seguintes instrumentos: dois manômetros digitais com escala de $0,00-10,00 \mathrm{kgf} \mathrm{cm}^{-2}$ e precisão de $0,01 \mathrm{kgf} \mathrm{cm}^{-2}$, previamente calibrados em um manômetro de peso morto com precisão de $0,1 \%$; um medidor de vazão magnético indutivo com precisão de $1 \%$ e um conjunto eletrobomba composto de duas unidades ligadas em série. Esses instrumentos integravam um circuito fechado, no qual a água pressurizada escoava através de tubulações de $25 \mathrm{~mm}$ de diâmetro.

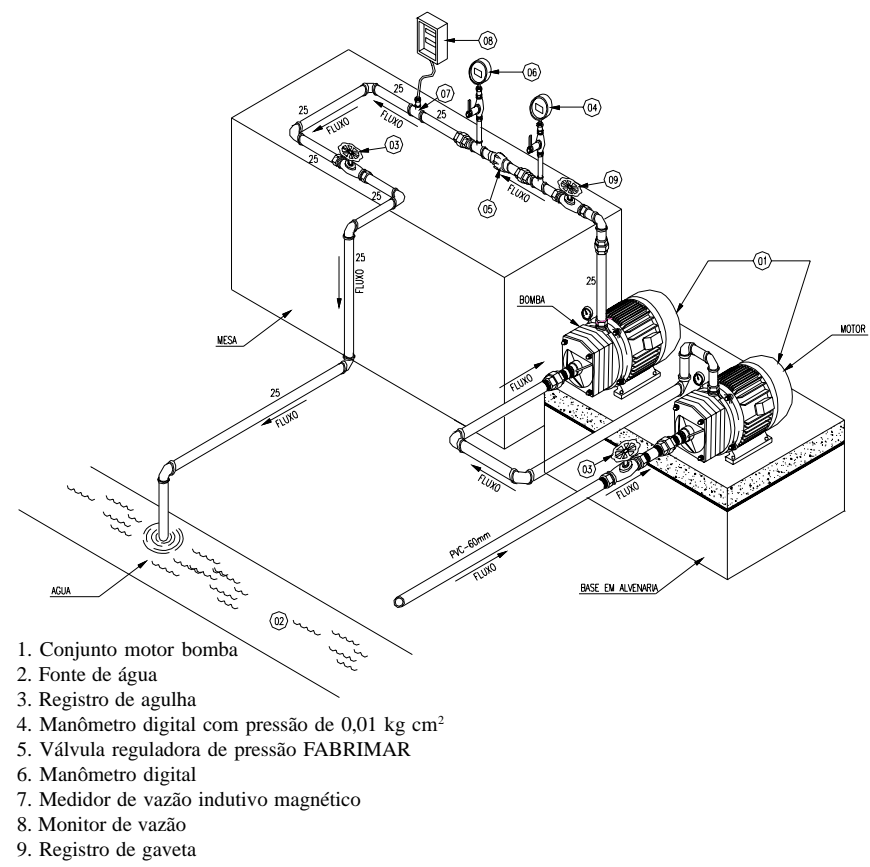

Figura 2. Esquema da bancada para o ensio dos reguladores

Os exemplares da amostra sem tempo de uso foram ensaiados nas seguintes vazões: $0,15,1,15,2,30,3,40$ e $4,50 \mathrm{~m}^{3} \mathrm{~h}^{-1}$. Permitiu-se uma variação máxima da vazão regulada de $\pm 2 \%$. As velocidades de referência utilizadas para esses exemplares, encontram-se na Tabela 1 e foram calculadas conforme especificado pela ISO (1993).

Os ensaios dos reguladores foram realizados em cada vazão (Q), utilizando-se 11 valores de pressão de entrada (PE): 68,5, 138, 172,4, 206,7, 275,1, 413,1, 481,7, 619,7, 688,2, 757,7 e $826,3 \mathrm{kPa}$. As pressões de saída (PS) foram medidas para pres-

Tabela 1. Vazões utilizadas no ensaio e as respectivas velocidades de referência

\begin{tabular}{llllll}
\hline A. Válvulas novas & & & & & \\
Vazão $\left(\mathrm{m}^{3} \mathrm{~h}^{-1}\right)$ & 1,15 & 2,30 & 3,40 & 4,50 & 0,15 \\
Vref $\left(\mathrm{m} \mathrm{s}^{-1}\right)$ & 0,13 & 1,02 & 2,03 & 3,01 & 3,98 \\
\hline B. Válvulas usadas & & & & & \\
Vazão $\left(\mathrm{m}^{3} \mathrm{~h}^{-1}\right)$ & 0,57 & 1,15 & 1,69 & 2,30 & 2,85 \\
Vref $\left(\mathrm{m} \mathrm{s}^{-1}\right)$ & 0,50 & 1,02 & 1,50 & 2,00 & 2,50 \\
\hline
\end{tabular}


Tabela 2. Características gerais das válvulas utilizadas

\begin{tabular}{|c|c|c|c|c|}
\hline Pivô & Proprietário & Total de Peças & $\begin{array}{l}\text { Tempo de } \\
\text { Uso }(\mathrm{h})\end{array}$ & Característica \\
\hline 1 & $\begin{array}{l}\text { Mauro Sakai } \\
\quad \text { (MS) }\end{array}$ & $\begin{array}{l}20 \text { reguladores de } 4 \text { vãos } \\
\text { diferentes }\end{array}$ & 2000 & Qualidade visualmente boa \\
\hline 2 & $\begin{array}{l}\text { Carlos Alberto Oliveira } \\
\text { (CAO) }\end{array}$ & $\begin{array}{l}25 \text { reguladores de } 5 \text { vãos } \\
\text { diferentes ( } 5 \text { reguladores/ } \\
\text { vão) }\end{array}$ & 2500 & $\begin{array}{l}\text { Qualidade visualmente boa, captação em } \\
\text { represa com algas }\end{array}$ \\
\hline 3 & $\begin{array}{l}\text { Itamarati Agropecuária S.A. } \\
\text { (ITA) }\end{array}$ & $\begin{array}{l}25 \text { reguladores de } 5 \text { vãos } \\
\text { diferentes ( } 5 \text { reguladores/ } \\
\text { vão) }\end{array}$ & 2500 & $\begin{array}{l}\text { Qualidade visualmente boa, captação em } \\
\text { represa limpa }\end{array}$ \\
\hline 4 & $\begin{array}{l}\text { Itamarati Agropecuária S.A. } \\
\text { (ITA) }\end{array}$ & $\begin{array}{l}25 \text { reguladores de } 5 \text { vãos } \\
\text { diferentes (5 reguladores/ } \\
\text { vão) }\end{array}$ & 6000 & $\begin{array}{l}\text { Qualidade visualmente boa, captação em } \\
\text { rio com um pouco de areia }\end{array}$ \\
\hline 5 & $\begin{array}{l}\text { Itamarati Agropecuária S.A. } \\
\text { (ITA) }\end{array}$ & $\begin{array}{l}25 \text { reguladores de } 5 \text { vãos } \\
\text { diferentes (5 reguladores/ } \\
\text { vão) }\end{array}$ & 8500 & $\begin{array}{l}\text { Qualidade visualmente regular, captação } \\
\text { em represa que recebeu chorume e vinhaça } \\
\text { durante certo tempo. Apresenta muita } \\
\text { corrosão no pivô. }\end{array}$ \\
\hline 6 & $\begin{array}{l}\text { Itamarati Agropecuária S.A. } \\
\text { (ITA) }\end{array}$ & $\begin{array}{l}25 \text { reguladores de } 5 \text { vãos } \\
\text { diferentes (5 reguladores/ } \\
\text { vão) }\end{array}$ & 9000 & $\begin{array}{l}\text { Qualidade visualmente boa, captação em } \\
\text { represa limpa }\end{array}$ \\
\hline 7 & $\begin{array}{l}\text { Mauro Sakai } \\
\quad \text { (MS) }\end{array}$ & $\begin{array}{l}25 \text { reguladores de } 5 \text { vãos } \\
\text { diferentes (5 reguladores/ } \\
\text { vão) }\end{array}$ & 10000 & $\begin{array}{l}\text { Qualidade visualmente péssima, negra, sem } \\
\text { vida, captada no Ribeirão do Jardim, a } 12 \\
\mathrm{~km} \text { à jusante da emissão de esgoto } \\
\text { "tratado" da cidade de Guaíra; captação } \\
\text { precária }\end{array}$ \\
\hline
\end{tabular}

sões de entrada gradualmente crescentes e, em seguida, decrescentes. Com a média desses valores, para uma amostra de tamanho 20, obteve-se a curva de regulação.

Os reguladores usados foram retirados em diferentes vãos de 7 pivôs, em 4 propriedades rurais, com captação de água de qualidade variada e com tempo de uso entre 2000 e $10000 \mathrm{~h}$, cujas características gerais estão apresentadas na Tabela 2.

Todos os reguladores usados foram submetidos a um processo de limpeza de detritos orgânicos e outros materiais sólidos que se acumularam no interior dos mesmos. Alguns modelos precisaram sofrer adaptações na saída para a realização do ensaio pois, originalmente, eram acoplados ao bocal, não se adaptando diretamente à bancada de ensaio.

Os ensaios dos reguladores usados foram realizados para 5 vazões apresentadas na Tabela 1B, com valores correspondentes aos de operação no campo: utilizaram-se 11 valores de pressão de entrada, conforme ensaio dos reguladores novos: $68,5,138,172,4,206,7,275,1,413,1,481,7,619,7,688,2,757,7 \mathrm{e}$ $826,3 \mathrm{kPa}$. As pressões de saída foram medidas para pressões de entrada gradualmente crescentes e decrescentes. Com a média desses valores, para uma amostra de tamanho 5 e para cada vazão, obteve-se a curva de regulação.

\section{RESULTADOS E DISCUSSÃO}

\section{Reguladores novos}

Os níveis de exatidão foram determinados para pressões de entrada superiores à pressão declarada de regulação, em todas as vazões testadas (Tabela 3). Verificou-se que, para atender às condições de um nível de exatidão $\mathrm{A}$, com as pressões de entrada especificadas na Tabela 3, o regulador de pressão deveria operar com vazão mínima superior a $0,15 \mathrm{~m}^{3} \mathrm{~h}^{-1}$ (velocidade de referência superior a $0,13 \mathrm{~m} \mathrm{~s}^{-1}$ ); de fato, a menor velocidade de referência indicada pela norma ISO (1993), para ensaio de reguladores de pressão, é $0,50 \mathrm{~m} \mathrm{~s}^{-1}$, o que corresponde, para o regulador ensaiado, à vazão de $0,57 \mathrm{~m}^{3} \mathrm{~h}^{-1}$, sendo que as pressões ensaiadas apresentam-se, nesta tabela, apenas a partir de 172,4 kPa.

Tabela 3. Desvios da pressão regulada medida durante o ensaio em relação à pressão declarada de regulação $(14 \mathrm{kPa})$

\begin{tabular}{crcccc}
\hline & \multicolumn{5}{c}{ Vazão $\left(\mathrm{m}^{3} \mathrm{~h}^{-1}\right)$} \\
\cline { 2 - 6 }$(\mathrm{kPa})$ & 0,15 & 1,15 & 2,30 & 3,40 & 4,5 \\
\cline { 2 - 6 } & \multicolumn{5}{c}{ Desvio $(\%)-$ Veja Eq. 1} \\
\hline 172,4 & 5,71 & 0,00 & $-2,86$ & $-6,43$ & $-14,29$ \\
206,7 & 8,57 & 1,43 & $-0,71$ & $-3,57$ & $-7,86$ \\
275,1 & 10,00 & 2,86 & 0,00 & $-2,86$ & $-6,43$ \\
413,1 & 10,00 & 2,86 & 0,71 & $-2,14$ & $-6,43$ \\
481,7 & 10,71 & 2,14 & 0,71 & $-2,14$ & $-6,43$ \\
619,7 & 10,00 & 2,14 & 0,71 & $-2,14$ & $-5,71$ \\
688,2 & 11,40 & 2,86 & 0,71 & $-2,14$ & - \\
757,7 & 13,60 & 5,71 & 2,14 & $-1,43$ & - \\
826,3 & 17,10 & 8,57 & 5,00 & - & - \\
\hline
\end{tabular}

Também, para a vazão máxima de ensaio de $4,50 \mathrm{~m}^{3} \mathrm{~h}^{-1}$ (velocidade de referência de $3,98 \mathrm{~m} \mathrm{~s}^{-1}$ ), o regulador de pressão só operou dentro dos limites especificados para o nível A de exatidão nas pressões de entrada de $206,7 \mathrm{kPa}$ até $619,7 \mathrm{kPa}$; já para as vazões de $1,15 \mathrm{~m}^{3} \mathrm{~h}^{-1}$ (velocidade de referência igual a $1,02 \mathrm{~m} \mathrm{~s}^{-1}$ ), 2,30 $\mathrm{m}^{3} \mathrm{~h}^{-1}$ (velocidade de referência $2,03 \mathrm{~m} \mathrm{~s}^{-1}$ ) e $3,40 \mathrm{~m}^{3} \mathrm{~h}^{-1}$ (velocidade de referência $3,01 \mathrm{~m} \mathrm{~s}^{-1}$ ) o regulador de pressão operou com nível $\mathrm{A}$ de exatidão em toda a faixa de pressão ensaiada. Deve-se observar que a maior velocidade de referência indicada pela ISO (1993), para ensaio de reguladores de pressão, é $2 \mathrm{~m} \mathrm{~s}^{-1}$, o que corresponde, para o regulador ensaiado, à vazão de $2,26 \mathrm{~m}^{3} \mathrm{~h}^{-1}$. O intervalo de regulação da pressão de entrada é aquele que inclui a pressão regulada no nível de exatidão especificado. 
Na Tabela 4 estão apresentados, resumidamente, os limites de regulação para a válvula reguladora de pressão, com pressão de regulação declarada de $137 \mathrm{kPa}$, considerando-se o nível de exatidão A. Nota-se que a velocidade de referência de $1,00 \mathrm{~m} \mathrm{~s}^{-1}$, recomendada pela norma, foi utilizada e que a pressão máxima não deve exceder a $619,7 \mathrm{kPa}$, pois ocorreu vibração da mola do regulador.

Tabela 4. Limites de regulação para o regulador de pressão Fabrimar, modelo EXACT-20, com pressão declarada de regulação de $137 \mathrm{kPa}$

\begin{tabular}{|c|c|c|c|}
\hline \multirow{2}{*}{$\begin{array}{l}\text { Vazão } \\
\left(\mathrm{m}^{3} \mathrm{~h}^{-1}\right)\end{array}$} & \multirow{2}{*}{$\begin{array}{c}\text { Velocidade } \\
\text { de Referência } \\
\quad\left(\mathrm{m} \mathrm{s}^{-1}\right)\end{array}$} & \multicolumn{2}{|c|}{$\begin{array}{c}\text { Intervalo } \\
\text { de Regulação }(\mathrm{kPa})\end{array}$} \\
\hline & & $\mathrm{PE}_{\text {mínima }}$ & $\mathrm{PE}_{\text {máxima }}$ \\
\hline 1,15 & 1,02 & 132,2 & $832,6^{1}$ \\
\hline 2,30 & 2,03 & 143,0 & $862,0^{1}$ \\
\hline 3,40 & 3,01 & 162,6 & $757,7^{2}$ \\
\hline 4,50 & 3,98 & 190,0 & $619,7^{2}$ \\
\hline
\end{tabular}

Valores extrapolados da curva de regulação

${ }^{2}$ Valores de pressão de entrada superiores a estes causaram vibração da mola do regulador

Obtidas as pressões médias de saída $\left(\mathrm{P}_{\mathrm{S}}\right)$ do regulador de pressão em função das pressões de entrada, para as cinco vazões ensaiadas, pode-se ajustar equações cujo modelo geral e seus coeficientes estão representados no cabeçalho da Tabela 5 , para cada vazão ensaiada. Verifica-se que os coeficientes de determinação $\left(\mathrm{R}^{2}\right)$ foram superiores a 0,99 para todos os ajustes. Na Tabela 6, encontram-se os valores médios de pressão de saída, determinados durante o ensaio.

De acordo com a norma ISO (1993), para a velocidade de referência de $1 \mathrm{~m} \mathrm{~s}^{-1}$ e dentro do intervalo de regulação, a variação da pressão regulada $\left(\mathrm{P}_{\mathrm{S}}\right)$ em relação à pressão de regulação declarada, não deverá ser superior a $10 \%$ para um regulador de pressão com nível de exatidão A, e não mais que $20 \%$ para um regulador com nível de exatidão B. Para esse regulador de pressão, as maiores variações foram 8,57 e 5,71\%, verificadas para as pressões de entrada de $826,2 \mathrm{kPa}$ e $138,0 \mathrm{kPa}$, respectivamente.

As curvas de regulação, ou curvas de desempenho, do regulador novo, são apresentadas na Figura 3, podendo-se observar sua característica em relação à velocidade de referência. Para a vazão de $0,15 \mathrm{~m}^{3} \mathrm{~h}^{-1}\left(0,13 \mathrm{~m} \mathrm{~s}^{-1}\right)$ o regulador não operou no limite de exatidão "A".
Tabela 6. Pressão média de saída determinada em função da pressão de entrada e da vazão para o regulador de pressão Fabrimar, modelo EXACT-20

\begin{tabular}{cccccc}
\hline \multirow{2}{*}{$\begin{array}{c}\text { Pressão de Entrada - PE } \\
(\mathrm{kPa})\end{array}$} & \multicolumn{5}{c}{ Vazão $\left(\mathrm{m}^{3} \mathrm{~h}^{-1}\right)$} \\
\cline { 2 - 6 } & 0,15 & 1,15 & 2,30 & 3,40 & 4,50 \\
\cline { 2 - 6 } & \multicolumn{5}{c}{ Pressão de Saída $(\mathrm{kPa})-\mathrm{PS}$} \\
\hline 68,5 & 6,7 & 6,3 & 6,0 & 5,3 & - \\
93,0 & - & - & - & - & 6,1 \\
138,0 & 13,7 & 13,2 & 12,3 & 11,1 & 9,7 \\
172,4 & 14,8 & 14,0 & 13,6 & 13,1 & 12,0 \\
206,7 & 15,2 & 14,2 & 13,9 & 13,5 & 12,9 \\
275,1 & 15,4 & 14,4 & 14,0 & 13,6 & 13,1 \\
413,1 & 15,4 & 14,4 & 14,1 & 13,7 & 13,1 \\
481,7 & 15,5 & 14,3 & 14,1 & 13,7 & 13,1 \\
619,7 & 15,4 & 14,3 & 14,1 & 13,7 & 13,2 \\
688,2 & 15,6 & 14,4 & 14,1 & 13,7 & - \\
757,7 & 15,9 & 14,8 & 14,3 & 13,8 & - \\
826,3 & 16,4 & 15,2 & 14,7 & - & - \\
\hline
\end{tabular}

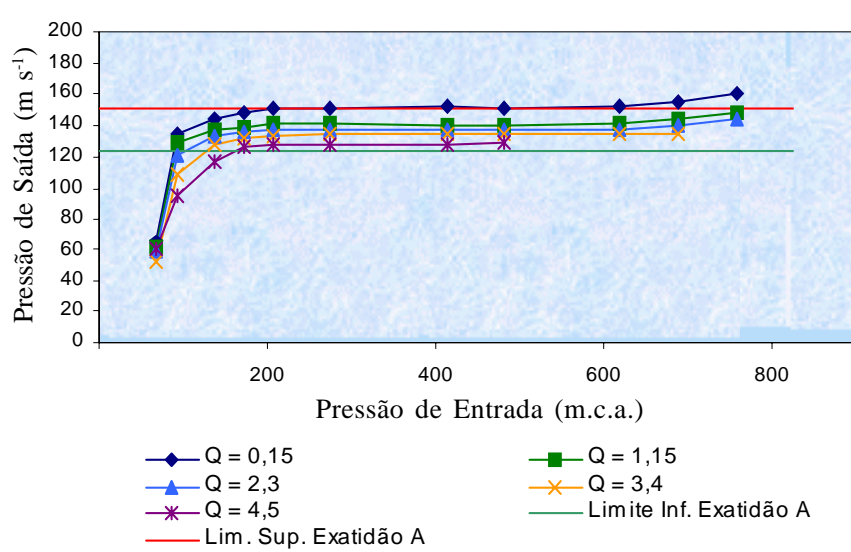

Figura 3. Pressão de saída média determinada em laboratório em função da pressão de entrada, em diferentes vazões $(\mathrm{Q}) \mathrm{em} \mathrm{m}^{3} \mathrm{~h}^{-1}$, para o regulador de pressão Fabrimar modelo Exact-20

Para as condições da pesquisa cujas velocidades de referência variaram de 0,13 a 4,0 $\mathrm{m} \mathrm{s}^{-1}$, tem-se que o número de Reynolds variou de 2600 a 80000 , caracterizando desde um regime laminar a um regime de fluxo turbulento. A redução na pressão de saída com o incremento na velocidade e, portanto, o número de Reynolds, pode ser explicada pela perda de carga, a qual, no regime turbulento, varia com aproximadamente a segunda potência da velocidade.

Tabela 5. Parâmetros das equações de regressão e coeficientes de determinação

\begin{tabular}{|c|c|c|c|c|c|}
\hline \multirow{2}{*}{$\begin{array}{l}\text { Coeficientes } \\
\text { do Modelo }\end{array}$} & \multicolumn{4}{|c|}{$P S=\frac{a+c P E+e P E^{2}+g P E^{3}}{1+b P E+d P E^{2}+f P E^{3}}$} & \\
\hline & \multicolumn{5}{|c|}{ Vazão $\left(\mathrm{m}^{3} \mathrm{~h}^{-1}\right)$} \\
\hline $\mathrm{a}$ & 0,0037690003 & 0,0034187616 & 0,0043258363 & 0,0028741926 & 0,013059465 \\
\hline $\mathrm{b}$ & $-0,078609485$ & $-0,0844377337$ & $-0,073259006$ & $-0,067924813$ & $-0,025205206$ \\
\hline $\mathrm{e}$ & 0,13522219 & 0,12174882 & 0,13967275 & 0,10528367 & $-0,076143997$ \\
\hline $\mathrm{f}$ & $-9,604067 \times 10^{-5}$ & $-9,375871 \times 10^{-5}$ & $-11,16614 \times 10^{-5}$ & $-6,396145 \times 10^{-5}$ & $25,06768 \times 10^{-5}$ \\
\hline $\mathrm{g}$ & $-0,0012493343$ & $-0,0011037857$ & $-0,0014012324$ & $-0,0007458271$ & 0,0035959409 \\
\hline $\mathrm{R}^{2}$ & 0,9985 & 0,9957 & 0,9984 & 0,9944 & 0,9984 \\
\hline Domínio $(\mathrm{kPa})$ & $68,5 \leq \mathrm{PE} \leq 688,7$ & $68,5 \leq \mathrm{PE} \leq 688,7$ & $68,5 \leq \mathrm{PE} \leq 688,7$ & $68,5 \leq \mathrm{PE} \leq 758,2$ & $93,0 \leq \mathrm{PE} \leq 620,1$ \\
\hline
\end{tabular}

$\mathrm{PE}=$ Pressão de entrada, em kPa; PS = Pressão de saída, em kPa 
Para segurança na operação do regulador de pressão Fabrimar modelo EXACT-20, sugere-se o seguinte domínio para o modelo que se apresenta no cabeçalho da Tabela 7 :

Para $\mathrm{Q} \leq 2,30 \mathrm{~m}^{3} \mathrm{~h}^{-1} \Rightarrow 70 \mathrm{kPa} \leq \mathrm{PE} \leq 844 \mathrm{kPa}$

Para 2,30 $\mathrm{m}^{3} \mathrm{~h}^{-1}<\mathrm{Q} \leq 3,40 \mathrm{~m}^{3} \mathrm{~h}^{-1}=>70 \mathrm{kPa} \leq \mathrm{PE} \leq 744 \mathrm{kPa}$

Para $3,40 \mathrm{~m}^{3} \mathrm{~h}^{-1}<\mathrm{Q} \leq 4,50 \mathrm{~m}^{3} \mathrm{~h}^{-1}=>95 \mathrm{kPa} \leq \mathrm{PE} \leq 633 \mathrm{kPa}$

Na Tabela 7, estão apresentados os modelos ajustados e os valores dos seus parâmetros. Verifica-se que a regressão explicou $99,16 \%$ da variação total dos dados e se observa os parâmetros que fazem parte do modelo para a obtenção da Pressão de Saída em função da Pressão de Entrada e da Vazão, onde se obteve um coeficiente de determinação.

Tabela 7. Pressão de saída (PS, kPa) em função da pressão de entrada $(\mathrm{PE}, \mathrm{kPa})$ e da vazão $\left(\mathrm{Q}, \mathrm{m}^{3} \mathrm{~h}^{-1}\right)$ para o regulador de pressão Fabrimar EXACT-20 (pressão declarada de regulação de $137,1 \mathrm{kPa}$ )

\begin{tabular}{lc}
\hline \multicolumn{2}{c}{ Modelo } \\
\hline \multicolumn{2}{c}{ PS $=\mathrm{a}+\mathrm{bQ}+\frac{\mathrm{c}}{1+\mathrm{e}^{\frac{\mathrm{d}-\mathrm{PE}}{\mathrm{f}}}}$} \\
\hline Parâmetros & Valor \\
\hline $\mathrm{a}$ & $-3,28060912$ \\
$\mathrm{~b}$ & $-0,53595555$ \\
$\mathrm{c}$ & 18,81388351 \\
$\mathrm{~d}$ & 6,219959147 \\
$\mathrm{e}($ Número de Nepper) & 2,71828183 \\
$\mathrm{~F}$ & 4,035721379 \\
\hline $\mathrm{R}^{2}$ (Coeficiente de determinação ajustado) & 0,9916 \\
\hline
\end{tabular}

\section{Reguladores com tempo de utilização}

Para as válvulas reguladoras usadas, analisaram-se pressões de saída em 5 velocidades de referência, variando de 0,5 a $2,5 \mathrm{~m} \mathrm{~s}^{-1}$. Durante os ensaios, foram observados dois tipos de vazamento:

Pelo orifício da câmara: o corpo da válvula reguladora de pressão possui uma câmara interna, Figura 2, necessária para a pressão atmosférica atuar; esta câmara é vedada por um anel de borracha que evita a passagem da água, o que é favorecido pela pressão que a mola exerce sobre o anel. $O$ desgaste da mola ou da borracha permitiu a entrada de água na câmara e o conseqüente vazamento pelo orifício existente na parte externa da válvula. Verificou-se, durante os ensaios, que o aumento da pressão de entrada proporcionava aumento do vazamento (quando este existia) mas, quando esta pressão chegava em torno dos 206,7 $\mathrm{kPa}$, o vazamento cessava. Em pressões decrescentes não ocorreu vazamento; possivelmente, a mola necessitava de certa pressão para começar a atuar no corpo da válvula, pressionando o anel de borracha que só assim conseguia evitar a passagem da água para a câmara;

Pela junta de vedação: neste experimento foram utilizadas válvulas de diversas idades, sendo que algumas, quando fabricadas, eram originalmente acopladas aos emissores, o que inviabilizaria o teste das mesmas nas condições da bancada existente no laboratório. Para viabilizar os ensaios, providen- ciou-se a remoção dos emissores, utilizando-se um "cabeçote" novo. Em alguns casos, essa nova conexão, com a junta de vedação antiga, não evitou por completo os vazamentos, principalmente quando algum parafuso proporcionava mais aperto. Esses vazamentos ocorriam sempre a baixas pressões de entrada crescentes.

Foram obtidos os valores médios das pressões de saída nas cinco velocidades ensaiadas, em função das pressões de entrada e do tempo de uso do regulador. As médias para os reguladores novos são apresentadas nas velocidades de 1,0 e $2,0 \mathrm{~m} \mathrm{~s}^{-1}$, que representam as principais velocidades definidas pela ISO (1993). O regulador da Fazenda Itamarati (ITA-2500 h) foi escolhido para a análise, representando o tempo de uso de 2500 h, ficando fora o regulador da Fazenda Campo Vitória $(\mathrm{CAO}-2500 \mathrm{~h})$ pois representava um mesmo número de horas de operação, mas possuía qualidade pior de água na sua captação e apresentou, também, curva pior. $\mathrm{Na}$ velocidade de 2,5 $\mathrm{m} \mathrm{s}^{-1}$, os reguladores novos e os da Fazenda Realeza (MS2000 h) não participaram da comparação das médias, porque não haviam sido avaliados nessa velocidade.

\section{Velocidade de referência de $0,5 \mathrm{~m} \mathrm{~s}^{-1}$}

Nesta velocidade, não houve diferença significativa, pelo teste de Tukey ao nível de 5\% de probabilidade, entre as pressões de saída dos reguladores com tempo de operação de 2000,2500 e 6000 h, até à pressão de entrada de $826,7 \mathrm{kPa}$ (Tabela 8A); entretanto, há diferenças significativas entre estes e aqueles que operaram por 8500,9000 e $10000 \mathrm{~h}$. As pressões de saída dos reguladores com 10000 h de operação, em geral, não diferiram das pressões dos reguladores com 8500 e $9000 \mathrm{~h}$ de operação nas diferentes pressões de entrada.

\section{Velocidade de referência de $1,0 \mathrm{~m} \mathrm{~s}^{-1}$}

Nesta velocidade, não houve diferença significativa, pelo teste de Tukey ao nível de 5\% de probabilidade, entre as pressões de saída dos reguladores novos, com tempo de operação de 2000, 2500 e $6000 \mathrm{~h}$, até à pressão de entrada de $826,3 \mathrm{kPa}$ (Tabela 8B), mas, há diferenças significativas entre o regulador novo e aqueles que operaram por 8500,9000 e 10000 $\mathrm{h}$, nas pressões de entrada superiores a $481,7 \mathrm{kPa}$. Abaixo desta pressão não ocorreram diferenças significativas entre as pressões de saída.

\section{Velocidade de referência de $1,5 \mathrm{~m} \mathrm{~s}^{-1}$}

Na velocidade de $1,5 \mathrm{~m} \mathrm{~s}^{-1}$ não houve diferença significativa, pelo teste de Tukey ao nível de 5\% de probabilidade, entre as pressões de saída dos reguladores com tempo de operação de $2000,2500,6000,8500$ e 9000 h, até à pressão de entrada de $757,7 \mathrm{kPa}$ (Tabela 8C); entretanto, há diferenças significativas entre estes e aqueles que operaram por $10000 \mathrm{~h}$, a partir de pressões de entrada superiores a $481,7 \mathrm{kPa}$.

\section{Velocidade de referência de $2,0 \mathrm{~m} \mathrm{~s}^{-1}$}

Nesta velocidade, não houve diferença significativa pelo teste de Tukey ao nível de 5\% de probabilidade, entre as pressões de saída dos reguladores novos, com tempo de operação de 2000, 2500, 6000 e $8500 \mathrm{~h}$, até à pressão de entrada de $826,3 \mathrm{kPa}$ (Tabela $8 \mathrm{D})$; porém, há diferenças significativas entre 
Tabela 8. Pressão de saída $(\mathrm{kPa})$ do regulador de pressão EXACT - 20, para diferentes pressões de entrada (kPa) e tempo de utilização (h), nas diferentes velocidades de referência

\begin{tabular}{|c|c|c|c|c|c|c|c|c|c|c|c|}
\hline \multirow{2}{*}{ Tempo (h) } & \multicolumn{11}{|c|}{ Pressão de Entrada (kPa) } \\
\hline & 68,5 & 138,0 & 172,4 & 206,7 & 275,1 & 413,1 & 481,7 & 619,7 & 688,2 & 757,7 & 826,3 \\
\hline \multicolumn{12}{|c|}{ Velocidade de referência $-0,5 \mathrm{~m} \mathrm{~s}^{-1}$} \\
\hline 0 & $\mathrm{X}$ & $\mathrm{X}$ & $\mathrm{X}$ & $\mathrm{X}$ & $\mathrm{X}$ & $\mathrm{X}$ & $\mathrm{X}$ & $\mathrm{X}$ & $\mathrm{X}$ & $\mathrm{X}$ & $\mathrm{X}$ \\
\hline 2000 & $73,2 \mathrm{BC} \mathrm{a}$ & $136,1 \mathrm{~A} \mathrm{~b}$ & $142,5 \mathrm{~A} \mathrm{bc}$ & $142,7 \mathrm{~A} \mathrm{bc}$ & $146,0 \mathrm{~A}$ bcd & $146,7 \mathrm{~A} \mathrm{bcd}$ & $146,9 \mathrm{~A} \mathrm{bcd}$ & $146,9 \mathrm{~A}$ bcd & $149,4 \mathrm{AB} c d$ & $152,2 \mathrm{~A} \mathrm{~cd}$ & $155,7 \mathrm{AB} \mathrm{d}$ \\
\hline 2500 & $71,6 \mathrm{~B} \mathrm{a}$ & $136,8 \mathrm{~A} \mathrm{~b}$ & $145,1 \mathrm{~A} \mathrm{bc}$ & $146,2 \mathrm{~A} \mathrm{bcd}$ & 147,1 A bcd & $146,9 \mathrm{~A} \mathrm{bcd}$ & $146,9 \mathrm{~A} \mathrm{bcd}$ & 146,9 A cd & $148,8 \mathrm{AB} \mathrm{cd}$ & $151,5 \mathrm{~A} \mathrm{~cd}$ & $155,7 \mathrm{AB} \mathrm{d}$ \\
\hline 6000 & $67,2 \mathrm{~A} \mathrm{a}$ & $133,7 \mathrm{~A} \mathrm{~b}$ & $141,5 \mathrm{~A}$ bc & $142,1 \mathrm{~A} \mathrm{~cd}$ & $142,5 \mathrm{~A}$ cd & $142,8 \mathrm{~A} \mathrm{~cd}$ & $143,2 \mathrm{~A} c d$ & 143,6 A cd & 144,4 A cd & $146,6 \mathrm{~A} \mathrm{~cd}$ & $150,2 \mathrm{~A} \mathrm{~d}$ \\
\hline 8500 & $74,4 \mathrm{C} \mathrm{a}$ & $135,7 \mathrm{~A} \mathrm{~b}$ & $143,5 \mathrm{~A} \mathrm{bc}$ & $145,5 \mathrm{~A} \mathrm{bc}$ & $143,9 \mathrm{~A} \mathrm{bc}$ & $144,9 \mathrm{~A} \mathrm{bc}$ & $146,5 \mathrm{~A} \mathrm{bc}$ & $149,9 \mathrm{~A} \mathrm{bc}$ & $152,2 \mathrm{ABC} b \mathrm{bc}$ & $155,7 \mathrm{AB}$ bc & $162,1 \mathrm{BC} \mathrm{c}$ \\
\hline 9000 & $72,3 \mathrm{BC} \mathrm{a}$ & $134,0 \mathrm{~A} \mathrm{~b}$ & 142,4 A bc & 142,7 A bc & 141,9 A bc & $141,8 \mathrm{~A} \mathrm{bc}$ & $145,5 \mathrm{~A} \mathrm{bcd}$ & 154,8 A cd & $161,2 \mathrm{BC} c d$ & $163,4 \mathrm{BC} \mathrm{d}$ & $164,3 \mathrm{BC} \mathrm{d}$ \\
\hline 10000 & $68,0 \mathrm{~A}$ a & $130,3 \mathrm{~A} \mathrm{~b}$ & 144,3 A bc & $151,1 \mathrm{~A} \mathrm{bc}$ & $145,8 \mathrm{~A} \mathrm{bc}$ & $131,9 \mathrm{~A} \mathrm{bc}$ & $139,0 \mathrm{~A} \mathrm{bc}$ & $156,1 \mathrm{~A} \mathrm{bc}$ & $163,3 \mathrm{C} \mathrm{bc}$ & $170,6 \mathrm{C} \mathrm{c}$ & $172,2 \mathrm{C} \mathrm{c}$ \\
\hline \multicolumn{12}{|c|}{ Velocidade de referência $-1,0 \mathrm{~m} \mathrm{~s}^{1}$} \\
\hline 0 & $63,4 \mathrm{~A} \mathrm{a}$ & $129,6 \mathrm{~A} \mathrm{a}$ & $137,5 \mathrm{~A} \mathrm{~b}$ & $139,0 \mathrm{~A} \mathrm{~b}$ & $140,9 \mathrm{~A} \mathrm{bc}$ & $141,5 \mathrm{~A} \mathrm{bc}$ & $140,2 \mathrm{~A} \mathrm{~b}$ & $140,4 \mathrm{~A} \mathrm{~b}$ & $141,1 \mathrm{~A} \mathrm{bc}$ & $144,7 \mathrm{~A} \mathrm{~cd}$ & $148,9 \mathrm{~A} \mathrm{~d}$ \\
\hline 2000 & $67,4 \mathrm{~B} \mathrm{a}$ & $132,3 \mathrm{~A} \mathrm{~b}$ & $140,3 \mathrm{~A} \mathrm{bc}$ & $141,7 \mathrm{~A} \mathrm{c}$ & $143,1 \mathrm{~A} \mathrm{c}$ & $145,7 \mathrm{~A} \mathrm{~cd}$ & $146,0 \mathrm{~A} \mathrm{~cd}$ & $145,9 \mathrm{AB} \mathrm{cd}$ & $146,2 \mathrm{AB} c d$ & $148,3 \mathrm{~A} \mathrm{~cd}$ & $151,7 \mathrm{Ad}$ \\
\hline 2500 & $67,7 \mathrm{~B} \mathrm{a}$ & $132,4 \mathrm{~A} \mathrm{~b}$ & 143,5 A bc & 144,3 A c & 144,8 A c & $145,0 \mathrm{~A} \mathrm{c}$ & 144,3 A c & $144,0 \mathrm{AB} \mathrm{c}$ & $145,9 \mathrm{AB} \mathrm{c}$ & 148,3 A c & $151,5 \mathrm{~A} \mathrm{c}$ \\
\hline 6000 & $69,2 \mathrm{~B} \mathrm{a}$ & $132,6 \mathrm{~A} \mathrm{~b}$ & $140,7 \mathrm{~A} \mathrm{bc}$ & $142,9 \mathrm{~A} \mathrm{bc}$ & $144,9 \mathrm{~A} \mathrm{bc}$ & $141,2 \mathrm{~A} \mathrm{bc}$ & $142,6 \mathrm{~A} \mathrm{bc}$ & $143,2 \mathrm{AB} b c$ & $144,7 \mathrm{AB} b c$ & $147,9 \mathrm{~A} \mathrm{bc}$ & $151,4 \mathrm{~A} \mathrm{c}$ \\
\hline 8500 & $68,6 \mathrm{~B} \mathrm{a}$ & $127,5 \mathrm{~A} \mathrm{~b}$ & $139,4 \mathrm{~A} \mathrm{bcd}$ & 144,0 A bcde & $136,0 \mathrm{~A} \mathrm{bc}$ & 142,9 A bcde & $145,8 \mathrm{~A}$ bcde & 152,9 B cde & $156,8 \mathrm{BC}$ cde & $161,3 \mathrm{~B} \mathrm{de}$ & $167,4 \mathrm{~B} \mathrm{e}$ \\
\hline 9000 & $67,6 \mathrm{~B} \mathrm{a}$ & $127,4 \mathrm{~A} \mathrm{~b}$ & $138,4 \mathrm{~A} \mathrm{bc}$ & $139,4 \mathrm{~A} \mathrm{bc}$ & $140,1 \mathrm{~A} \mathrm{bcd}$ & $139,0 \mathrm{~A} \mathrm{bc}$ & $139,3 \mathrm{~A} \mathrm{bc}$ & $148,2 \mathrm{AB}$ bcd & $155,4 \mathrm{BC}$ bcd & $163,0 \mathrm{~B} \mathrm{~cd}$ & $170,3 \mathrm{~B} \mathrm{~d}$ \\
\hline 10000 & $68,2 \mathrm{~B} \mathrm{a}$ & $131,3 \mathrm{~A} \mathrm{~b}$ & $143,3 \mathrm{~A} \mathrm{bc}$ & $145,1 \mathrm{~A} \mathrm{bc}$ & $144,7 \mathrm{~A} \mathrm{bc}$ & $142,5 \mathrm{~A} \mathrm{bc}$ & $144,9 \mathrm{~A} \mathrm{bc}$ & 154,2 B bcd & $160,8 \mathrm{C} \mathrm{cd}$ & $167,1 \mathrm{~B} \mathrm{~cd}$ & $176,3 \mathrm{~B} \mathrm{~d}$ \\
\hline
\end{tabular}

C. Velocidade de referência $-1,5 \mathrm{~m} \mathrm{~s}^{-1}$

$\begin{array}{ccc}0 & \mathrm{X} & \mathrm{X} \\ 2000 & 60,2 \mathrm{~A} \mathrm{a} & 128,3 \mathrm{~A} \mathrm{~b} \\ 2500 & 61,1 \mathrm{AB} \text { a } & 127,2 \mathrm{~A} \mathrm{~b} \\ 6000 & 64,3 \mathrm{C} \mathrm{a} & 128,9 \mathrm{~A} \mathrm{~b} \\ 8500 & 62,7 \mathrm{BC} \mathrm{a} & 127,1 \mathrm{~A} \mathrm{~b} \\ 9000 & 62,7 \mathrm{BC} \mathrm{a} & 126,3 \mathrm{~A} \mathrm{~b} \\ 10000 & 63,0 \mathrm{C} \mathrm{a} & 121,1 \mathrm{~A} \mathrm{~b}\end{array}$

D. Velocidade de referência $-2,0 \mathrm{~m} \mathrm{~s}^{-1}$

\begin{tabular}{|c|c|c|c|c|c|c|c|c|c|c|c|}
\hline 0 & $58,7 \mathrm{C} \mathrm{a}$ & $120,6 \mathrm{AB} b$ & $133,4 \mathrm{~A} \mathrm{c}$ & $135,5 \mathrm{~A} \mathrm{~cd}$ & 137,2 A cde & 137,6 A cde & $138,1 \mathrm{~A} \mathrm{de}$ & $137,6 \mathrm{Ade}$ & $138,2 \mathrm{~A} \mathrm{de}$ & $139,8 \mathrm{Ae}$ & $144,1 \mathrm{Af}$ \\
\hline 2000 & $55,7 \mathrm{AB}$ a & $121,2 \mathrm{AB} B$ & 133,6 A c & $137,0 \mathrm{~A} \mathrm{~cd}$ & $139,4 \mathrm{AB}$ cde & 140,8 A cde & 140,5 A cde & $140,5 \mathrm{AB}$ cde & $143,0 \mathrm{AB}$ cde & 146,6 Ade & $149,4 \mathrm{~A} \mathrm{e}$ \\
\hline 6000 & $58,9 \mathrm{BC} \mathrm{a}$ & 124,6 B b & $135,2 \mathrm{~A} \mathrm{c}$ & $136,6 \mathrm{~A} \mathrm{c}$ & $136,6 \mathrm{~A} \mathrm{c}$ & $136,6 \mathrm{~A} \mathrm{c}$ & $136,7 \mathrm{~A} \mathrm{c}$ & 136,8 A c & $137,3 \mathrm{~A} \mathrm{c}$ & $139,1 \mathrm{Ac}$ & $142,8 \mathrm{~A} \mathrm{c}$ \\
\hline 8500 & $59,9 \mathrm{C} \mathrm{a}$ & $122,6 \mathrm{AB} b$ & $135,1 \mathrm{~A} \mathrm{bc}$ & 138,3 A c & $138,2 \mathrm{AB} \mathrm{c}$ & $137,5 \mathrm{~A} \mathrm{c}$ & 137,4 A c & $137,5 \mathrm{~A} \mathrm{c}$ & 138,0 A c & $142,0 \mathrm{Ac}$ & $145,8 \mathrm{~A} \mathrm{c}$ \\
\hline 10000 & $58,3 \mathrm{BC} \mathrm{a}$ & $117,1 \mathrm{~A} \mathrm{~b}$ & $132,1 \mathrm{~A} \mathrm{~cd}$ & $144,2 \mathrm{~A} \mathrm{bcd}$ & $169,4 \mathrm{AB}$ bcd & $141,4 \mathrm{~A}$ bcd & 141,6 A bcd & $163,4 \mathrm{C} \mathrm{bcd}$ & $171,0 \mathrm{C} \mathrm{cd}$ & $178,3 \mathrm{~B} \mathrm{~cd}$ & $185,7 \mathrm{C} \mathrm{d}$ \\
\hline
\end{tabular}

E. Velocidade de referência $-2,5 \mathrm{~m} \mathrm{~s}^{-1}$

\begin{tabular}{|c|c|c|c|c|c|c|c|c|c|c|c|}
\hline 0 & $\mathrm{X}$ & $\mathrm{X}$ & $X$ & $\mathrm{X}$ & $\mathrm{X}$ & $\mathrm{X}$ & $\mathrm{X}$ & $\mathrm{X}$ & $\mathrm{X}$ & $\mathrm{X}$ & $X$ \\
\hline 6000 & $53,8 \mathrm{CD}$ & $120,3 \mathrm{AB}$ & $134,1 \mathrm{~A} \mathrm{c}$ & 134,2 A c & $135,0 \mathrm{~A} \mathrm{c}$ & $134,3 \mathrm{~A} \mathrm{c}$ & $133,5 \mathrm{~A} \mathrm{c}$ & $133,6 \mathrm{~A}$ & $135,0 \mathrm{~A} \mathrm{c}$ & $138,3 \mathrm{~A} \mathrm{c}$ & $141,9 \mathrm{~A} \mathrm{c}$ \\
\hline 8500 & $48,6 \mathrm{~A}$ & $115,8 \mathrm{AB}$ & $132,6 \mathrm{~A} \mathrm{c}$ & $134,5 \mathrm{~A} \mathrm{~cd}$ & $134,1 \mathrm{~A} \mathrm{~cd}$ & $133,6 \mathrm{~A} \mathrm{~cd}$ & $133,7 \mathrm{~A} \mathrm{~cd}$ & $135,1 \mathrm{~A} \mathrm{~cd}$ & $137,9 \mathrm{AB} \mathrm{cd}$ & $140,3 \mathrm{AB}$ cd & 144,3 A d \\
\hline 10000 & $54,3 \mathrm{D}$ & 114,7 A & $131,4 \mathrm{~A} \mathrm{bc}$ & $141,4 \mathrm{~A} \mathrm{bcd}$ & 149,9 A bcd & $139,8 \mathrm{~A} \mathrm{bcd}$ & $147,5 \mathrm{~A}$ & 167,2 A cde & $174,5 \mathrm{C}$ de & $182,3 \mathrm{D} \mathrm{e}$ & $186,8 \mathrm{C} \mathrm{e}$ \\
\hline
\end{tabular}

\section{$\mathrm{X}$}

$142,4 \mathrm{Ac}$

$138,0 \mathrm{~A} \mathrm{c}$

$140,4 \mathrm{~A} \mathrm{bc}$

$\mathrm{X}$

139,5 A bcd

145,6 Ac

140,3 A cd

143,0 Abc

139,0 Abc

X

$141,3 \mathrm{~A} \mathrm{bc}$

$147,0 \mathrm{~A} \mathrm{c}$

$141,8 \mathrm{~A} \mathrm{~cd}$

$140,4 \mathrm{~A} \mathrm{bc}$

$\mathrm{X}$

$142,8 \mathrm{~A} \mathrm{bcd}$

$\mathrm{X}$

147,0 A c

$141,8 \mathrm{~A} \mathrm{~cd}$

$139,8 \mathrm{~A} \mathrm{bc}$

142,6 A bcd

146,7 A c

\section{$\mathrm{X}$}

141,6 A cde

$147,2 \mathrm{AB}$ c

140,9 A cd

$141,3 \mathrm{~A} \mathrm{~cd}$

$140,4 \mathrm{~A} \mathrm{bc}$

$148,3 \mathrm{AB}$

$\mathrm{X}$

143,1 A cde

149,7 A c

141,9 A cd

$142,0 \mathrm{~A} \mathrm{bc}$

$151,1 \mathrm{Acd}$

$\mathrm{X}$

15,9 A de

$144,9 \mathrm{~A} \mathrm{~cd}$

144,3 A bc

$155,2 \mathrm{~A} \mathrm{~cd}$ 174,8 B de 
A.
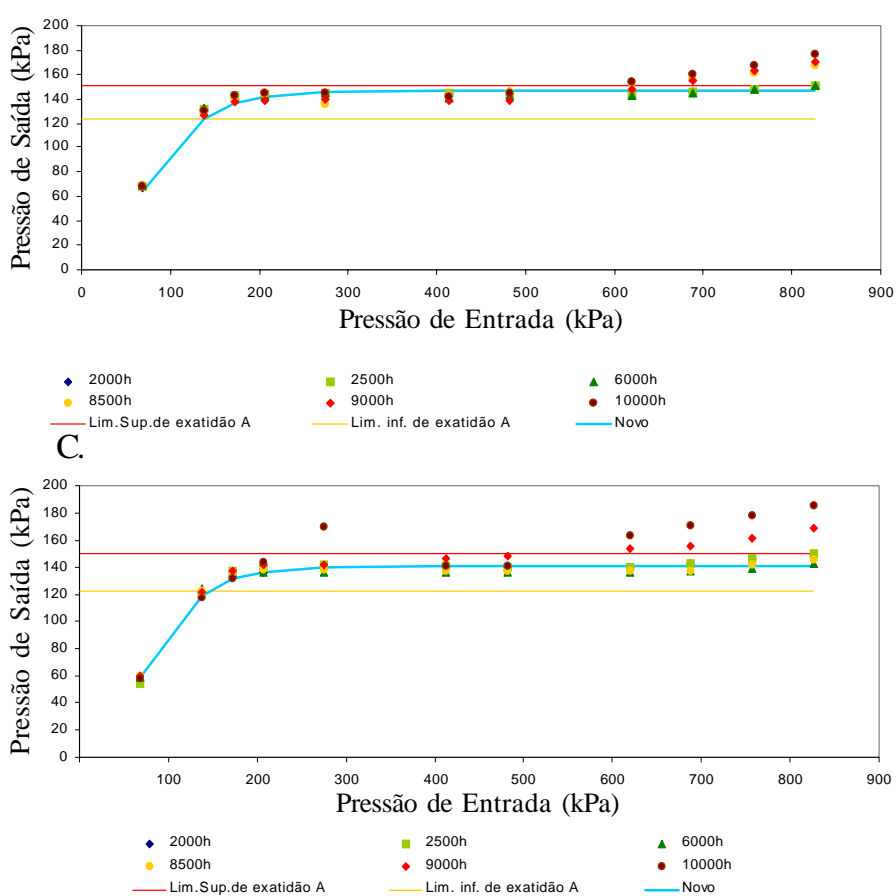

Figura 5. Comparação entre as curvas de desempenho dos reguladores novos e usados, avaliados na velocidade de referência de $1,0(\mathrm{~A}), 1,5(\mathrm{~B}), 2,0(\mathrm{C}) \mathrm{e} 2,5 \mathrm{~m} \mathrm{~s}^{-1}(\mathrm{D})$

o regulador novo e aqueles que operaram durante 9000 e 10000 $\mathrm{h}$, nas pressões de entrada superiores a 481,7 kPa. Abaixo desta pres-são, não ocorreram diferenças significativas entre as pressões de saída, mas somente para valores de pressão de entrada superiores a $619,7 \mathrm{kPa}$, os reguladores de pressão com 9000 e 10000 h de operação apresentaram diferenças significativas nas pressões de saída.

\section{Velocidade de referência de $2,5 \mathrm{~m} \mathrm{~s}^{-1}$}

Na velocidade de $2,5 \mathrm{~m} \mathrm{~s}^{-1}$, os resultados da análise estatística mostraram pouca consistência na tendência dos dados obtidos nos ensaios. Na Tabela 8E, observa-se que se pode estabelecer os seguintes grupos de reguladores de pressão, que não apresentaram diferenças significativas nas pressões de saída, em função do tempo de operação: reguladores com tempo de operação de 6000 e $8500 \mathrm{~h}$; reguladores com tempos de operação de 2500 e 9000 h. Um resultado consistente é a diferença estatística entre reguladores com tempo de operação de 10000 h e os demais.

Os pontos correspondentes às curvas de desempenho dos reguladores usados foram plotados em gráficos para comparálos com a curva do regulador novo obtida pelo modelo apresentado na Tabela 6 , nas velocidades de 1,0, 1,5, 2,0 e 2,5 $\mathrm{m} \mathrm{s}^{-1}$. Nas Figuras 5A, B, C e D podem ser observadas as diferenças nas pressões de entradas relativas a essas comparações. Para a confecção destes gráficos escolheu-se para a categoria de $2500 \mathrm{~h}$ de uso, apenas o regulador ITA, pois apresentou melhor qualidade que o M.S.

Observa-se que, na velocidade de referência de $1,0 \mathrm{~m} \mathrm{~s}^{-1}$, os reguladores de 8500,9000 e $10000 \mathrm{~h}$, a partir da pressão de entrada de $688,2 \mathrm{kPa}$, extrapolaram o limite do intervalo de regulação do nível exatidão "A", sendo que os demais regula-
B.

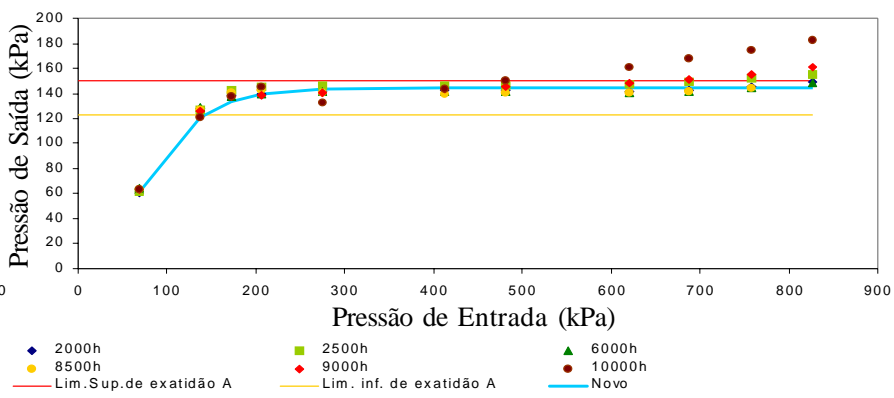

D.

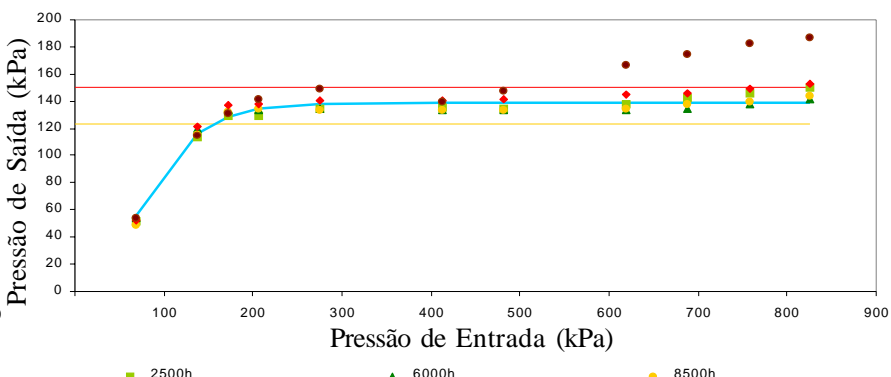

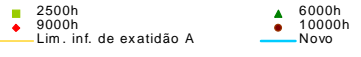

dores não apresentaram variações significativas em relação ao novo, mantendo-se dentro daquele limite.

Na velocidade de $1,5 \mathrm{~m} \mathrm{~s}^{-1}$, a partir de $619,7 \mathrm{kPa}$ de pressão de entrada, houve grande variação da pressão de saída para os reguladores 9000 e $10000 \mathrm{~h}$ em relação ao novo, sendo que os demais permaneceram dentro do limite de regulação recomendado.

Para a velocidade de referência de $2,0 \mathrm{~m} \mathrm{~s}^{-1}$, o regulador de $10000 \mathrm{~h}$ não regulou, pois apresentou grande variação na pressão de saída em relação ao regulador novo. O regulador de $9000 \mathrm{~h}$ começou a extrapolar o limite de exatidão "A" na pressão de entrada de $619,7 \mathrm{kPa}$.

Finalmente, na velocidade de $2,5 \mathrm{~m} \mathrm{~s}^{-1}$, apenas o regulador de pressão de $10000 \mathrm{~h}$ apresentou a curva de regulação mais distante da curva do regulador de pressão novo, sendo que os demais permaneceram no nível de exatidão "A".

\section{CONCLUSÕES}

Considerando-se os resultados obtidos e as análises realizadas com a válvula reguladora de pressão Fabrimar, modelo EXACT-20 - 3/4" FF, conclui-se que:

1. A válvula reguladora de pressão nova satisfaz as condições exigidas para ser classificada como regulador de pressão com nível de exatidão $\mathrm{A}$, nas velocidades de referência de $0,5 \mathrm{~m} \mathrm{~s}^{-1}$ (vazão de $0,57 \mathrm{~m}^{3} \mathrm{~h}^{-1}$ ) até $4,0 \mathrm{~m} \mathrm{~s}^{-1}$ (vazão de $4,50 \mathrm{~m}^{3} \mathrm{~h}^{-1}$ ).

2. O desempenho hidráulico do regulador de pressão, com tempo de uso até $6000 \mathrm{~h}$, não diferiu do desempenho do regulador novo, para pressões de entrada até $826,7 \mathrm{kPa}$ e velocidades de referência até $2,5 \mathrm{~m} \mathrm{~s}^{-1}$.

3. O desempenho hidráulico dos reguladores de pressão com tempos de uso iguais ou superiores a $8500 \mathrm{~h}$ não se manteve semelhante ao do novo, especialmente para pressões de entrada superiores a $481,9 \mathrm{kPa}$. 


\section{LITERATURA CITADA}

Benami, A.; Ofen, A. Irrigation engineering. Haifa: Irrigation Engineering Scientific Pubications (IESP), 1984, 252p.

Frizzone, J.A. Irrigação por aspersão: uniformidade e eficiência. Piracicaba: ESALQ, Dept. Eng. Rural, 1992. 53p. Série Didática, 3

Frizzone, J.A.; Botrel, T.A. Ensaio \& certificação de sistemas e equipamentos de irrigação. In: Mialhe, L.G. Máquinas Agrícolas: Ensaios \& Certificação. Piracicaba: Fundação de Estudos Agrários Luiz de Queiroz, 1996. 722p.

ISO - International Organization For Standardization, ISO 10522, Agricultural irrigation equipment - Direct-acting pressureregulating valves, Geneve, 1993, 11p.
Keller, J.; Bliesner, R.D. Sprinkler and trickle irrigation. New York: Avi Book, 1990, 652p.

Klar, A.E.; Santana, R. de C.; Duroha, C. Evaluation center pivot systems using new and old sprays and pressure regulators. IRRIGA, Botucatu, v.6, n.1, 2001.

Tarjuelo, J.M.M.B. El Riego por Aspersion y su Tecnologia, Madrid: Mundi Prensa, 1994, 491p.

von Bernuth, R.D.; Baird, D. Characterizing pressure regulator performance. Transactions of the ASAE, St. Joseph, v.33, n.1, p.145-150, 1990.

Zaggo, S.P.; Colombo, A.; Gil, O.F. Desempenho de válvulas reguladoras de pressão utilizadas em sistemas pivô central, Congresso Brasileiro de Engenharia Agrícola, 19, Anais, v.1, p.360-379, Piracicaba, SBEA, 1990. 